\title{
The Association Between Emotional and Behavioral Problems in Children with Autism Spectrum Disorder and Psychological Distress in Their Parents: A Systematic Review and Meta-analysis
}

\author{
Isabel Yorke $^{1}$ (D) $\cdot$ Pippa White $^{1} \cdot$ Amelia Weston $^{2} \cdot$ Monica Rafla $^{1} \cdot$ Tony Charman $^{1} \cdot$ Emily Simonoff $^{1}$
}

Published online: 18 May 2018

(c) The Author(s) 2018

\begin{abstract}
This review (Prospero Registration Number: CRD42017057915) aimed to systematically identify and summarize existing research on the relationship between additional emotional and behavioral problems (EBP) in children with autism, and parenting stress (PS) and mental health problems (MHP) in their parents. Sixty-seven studies met criteria for inclusion in the review, 61 of which were included in the meta-analysis. Pooled correlation coefficients were in the low to moderate range ( $\vec{r}=-.21$ to .43). Some evidence for moderation by measurement characteristics was found. Narrative review of concurrent adjusted associations showed some evidence for shared relationships with other factors, most notably ASD severity and parent perception of own parenting. Longitudinal studies showed mixed evidence for bidirectional predictive relationships between child EBP and parent psychological distress variables.
\end{abstract}

Keywords Autism spectrum disorder · Additional psychopathology · Emotional and behavioral problems · Mental health · Parenting stress $\cdot$ Psychological distress

\section{Introduction}

Research in the general population has established wellcharacterized associations between child psychopathology and elements of parental psychological distress, including parenting stress (PS) (Crnic et al. 2005) and mental health problems (MHP) (Goodman et al. 2011). Population-based research has shown PS (Keyser et al. 2017) and MHP (Ford et al. 2004) to retain a significant association with child psychopathology after adjusting for other family, child and contextual factors. Longitudinal research has shown support for reciprocal predictive relationships between child emotional and behavioral problems (EBP) and parent psychological

Electronic supplementary material The online version of this article (https://doi.org/10.1007/s10803-018-3605-y) contains supplementary material, which is available to authorized users.

Isabel Yorke

isabel.yorke@kcl.ac.uk

1 Institute of Psychiatry, Psychology \& Neuroscience, King's College London, 16 De Crespigny Park, London SE5 8AF, UK

2 Department of Psychology, University of Bath, Bath, UK wellbeing, for example depression (Bagner et al. 2013) and in certain conditions, PS (Stone et al. 2016). Although it is plausible that a shared genetic component may account for the correlation between psychopathology in children and their parents, research conducted using genetically sensitive designs has evidenced an important role for environment in the intergenerational association of anxiety (Eley et al. 2015), depression (McAdams et al. 2015) and conduct problems (D'Onofrio et al. 2007).

Children with autism spectrum disorder (ASD) (American Psychiatric Association 2013) are highly likely to meet criteria for additional mental health disorders (de Bruin et al. 2007; Salazar et al. 2015; Simonoff et al. 2008). These commonly take the form of both internalizing (e.g. anxiety or depressed mood) and externalizing (e.g. conduct problems, oppositional behavior or hyperactivity) problems. They have been identified as a source of particular difficulty and unmet need for individuals and their families (Cadman et al. 2012; Kring et al. 2008). Some previous research indicates that such EBP are more closely related to family functioning (McStay et al. 2014b; Pozo et al. 2014) and parent wellbeing (Vasilopoulou and Nisbet 2016) than is core ASD symptom severity. 
Increased psychological distress has also been demonstrated in parents of children with ASD. Parents of children with ASD experience high rates of PS, even in comparison to parents of children with other developmental disorders, intellectual difficulties and physical disabilities (Estes et al. 2009; Hayes and Watson 2013). Increased rates of MHP are also seen both before and after the child's birth (Bolton et al. 1998; Jokiranta et al. 2013). Research in the general population has shown that PS and MHP are related but separable phenomena, with each tending to increase vulnerability to the other (Deater-Deckard 2004). For example, underlying susceptibility to poor mental health may compromise ability to cope with parenting demands. Equally, intense parenting challenges may initiate the development of psychopathology. In parents of children with ASD specifically, it is plausible for both these processes to be at work. Given the heightened rates of psychopathology in both children with ASD and their parents, and the potential for each to exacerbate the other, investigating their association in these families essential.

A growing body of research has investigated PS and MHP for their candidacy as contributors to, or consequences of, additional EBP in children with ASD. The purpose of this systematic review and meta-analysis is to identify and synthesize such research. Most has been cross-sectional, seeking to identify factors that account for variance in the rates and severity of parent MHP, PS or child EBP. A plethora of other child, parent and contextual factors has been investigated for their involvement in the relationships of interest, meaning that comparison across studies is not straightforward. In order to summarize such research, the current study includes a meta-analysis of unadjusted concurrent relationships and narrative review of concurrent relationships adjusted for other factors. Longitudinal research is also reviewed narratively.

This review had four main research objectives. Firstly, we aimed to establish the magnitude of the concurrent associations between child EBP and parent psychological distress variables (MHP and PS) in families of children with ASD. Based on research in the general population (Costa et al. 2006; Goodman et al. 2011; Crnic et al. 2005), meta-analysis was expected to reveal significant pooled concurrent associations of small to moderate magnitude. Although the relationships between parent psychological distress variables and child EBP appear similar in magnitude across externalizing and internalizing problems in the general population (e.g. Goodman et al. 2011), evidence nevertheless supports an etiological distinction between the two (Cosgrove et al. 2011). We therefore opted to run separate analyses for child externalizing and internalizing problems, in addition to those for total EBP.

Secondly, we aimed to explore which methodological factors explain variation across studies in the magnitude of these relationships. Goodman and colleagues (2011) found in a meta-analysis of general population studies that the relationship between maternal depression and child EBP was statistically moderated by various methodological factors including mean age of the child sample, clinical versus community recruitment of mothers and informant for child EBP. We correspondingly aimed to investigate these factors as potential moderators in our analyses. Of particular interest was the effect of informant, since the majority of the relevant literature relies on parent-report questionnaire measures for both child and parent variables. This approach may inflate associations due to shared method biases (Podsakoff et al. 2003) and the possible tendency for distressed parents to rate their children's problems as more severe (Najman et al. 2000). We therefore planned separate analyses for effect sizes deriving from a rating of child EBP by the index parent, or those by an alternative informant.

Our third objective was to ascertain whether the associations of interest maintain once other child factors (e.g. ASD severity; IQ), parent factors (e.g. coping style; parenting behaviors; social support) and contextual factors (e.g. family income; socio-economic status) are statistically accounted for. In order to guide future mental health research in families of children with ASD, it is important to (a) establish whether other factors can fully or partially account for the relationship between parent and child psychological well-being and (b) identify other factors which may play a role in the architecture of these relationships. Although studies conducted at single time-points cannot inform us as to causality or the temporal precedence of one factor over another (Kraemer et al. 2001), they may guide us as to which variables are most likely to be involved, and therefore which should be selected for investigation in longitudinal and intervention research.

Our final objective was to examine the literature for evidence as to predictive (longitudinal) relationships between child EBP and parental MHP or PS in this population. Specifically, we wished to establish whether child EBP predicts elements of parent psychological distress, whether the opposite is true, or whether both are risk factors for each other. To meet this objective, a descriptive review was planned. Although quantitative analysis was to be performed if available data allowed it, in fact they did not. Based on research in the general population, we expected to find bidirectional predictive relationships, i.e. earlier parent MHP and PS predict later child EBP and vice versa (Bagner et al. 2013; Neece et al. 2012; Nicholson et al. 2011; Zadeh et al. 2010).

\section{Methods}

\section{Search Procedures}

Search terms were initially run in the Cochrane Database of Systematic Reviews to evaluate coverage of the topic 
of interest by existing reviews. Upon finding no equivalent reviews, the same search was then conducted in the Cochrane Library, Medline, PsycINFO, PsycArticles, Embase and Web of Science databases. Search strategies included keywords pertaining to ASD (including "autis*", "Asperger*" and "pervasive developmental disorder") and additional EBP (including "psychiatr*, "psychopatholog*", "externali\$ing", "internali\$ing”, "behavio $\$ r$ problem*" and "emotional problem*"). These terms were combined with terms for caregiver psychological distress, produced by searching for "parent*", "mother*", "maternal", "father*", "paternal", or "caregiver*" within two words of "stress*", "distress*", "psychopatholog*", "psychiatr*", "mental health" or "mental disorder*". Subject headings were used according to the capabilities of each database. Complete search strategies are provided in the supplemental material. Searches were conducted on three occasions: 12th April 2016, 6th September 2016 and 9th April 2017. Email alerts were set up to capture relevant literature published after these dates. Reference lists of included studies were searched manually to identify any eligible studies missed by the search strategy. This study was added to the PROSPERO registry at the study screening stage (CRD42017057915).

\section{Inclusion Criteria}

This review included original articles published in or after the year 2000 in the English language that met the following criteria.

\section{Participant Characteristics}

Study samples were required to include at least 20 individuals with a reported clinical diagnosis of ASD, and their parents or main unpaid caregivers (hereafter, parents). The minimum mean age of the children was 3 years ( 36 months) and the maximum mean age was 21 years. There was also a maximum upper age limit of 25 years, in order to conserve homogeneity (i.e. samples whose age range extended beyond 25 years were excluded). There were no specifications regarding the age of the parents. Studies with multiple time-points were included provided a relevant analysis was conducted for at least one time within these age boundaries. If analysis at more than one time-point met criteria, the earliest of these was included in the meta-analyses of concurrent association. In the case of multiple studies reporting on the same sample, the study that reported the most relevant information was selected for inclusion in the analysis, whilst the others were excluded.

\section{Measurement Requirements}

Studies were required to include at least one quantitative measurement of additional EBP in young people with diagnosed ASD. This measure was required primarily to tap common EBP. Measures containing several items pertaining to ASD-like behaviors were permitted, provided these did not form a majority. Secondly, studies were required to include at least one quantitative self-report measure of caregiver PS or common MHP, e.g. anxiety and/or depression. Quality of life measures often include a mental health component; however, this concept is not necessarily the same as MHP. Such studies have been reviewed elsewhere (Vasilopoulou and Nisbet 2016), thus they were not included here.

\section{Analysis Requirements}

Studies reporting results of at least one statistical analysis of association between the two required measures, within the sample of participants meeting above criteria, were eligible. To be included in the meta-analysis component, an unadjusted (zero-order) correlation coefficient was required (ideally, Pearson's $r$ ). In studies that conducted a relevant analysis, but did not provide Pearson's $r$, various procedures were used to obtain or impute an effect size. Firstly, authors were contacted and asked to supply Pearson's $r$. Failing this, we imputed Pearson's $r$ from Spearman's rho, phi coefficients t-tests or Chi square values using the Practical MetaAnalysis Effect Size Calculator (Wilson 2001). This was done for 5 studies (8.20\%). In the case of one study (Bromley et al. 2004), effect sizes were only reported for significant associations, and those for non-significant associations were not recoverable. In such cases, a Pearson's $r$ value of .00 was imputed. Standardized regression coefficients (betas) for simple regressions (with only one predictor) were used in place of Pearson's $r$ for several studies to minimize missing data. Studies that reported only statistics for adjusted relationships (e.g. multiple regressions, partial correlations, ANCOVA) were excluded from the meta-analysis component. Such analyses were reviewed narratively to address Objective 3.

\section{Exclusion Criteria}

Samples in which the majority of participants had nonidiopathic ASD (e.g. that attributable to a known genetic syndrome) were excluded, since such syndromes tend to have their own characteristic profiles of symptoms (Glennon et al. 2017), including EBP. In samples comprising children with various developmental disorders, those with less than 50\% diagnosed with ASD were excluded. Studies in which the majority of participating children lived away from the family home were excluded. 


\section{Study Selection and Data Extraction}

Studies identified in the search were reviewed for inclusion by IY. Just over $25 \%$ of the 414 articles which progressed to screening by full text $(n=106)$ were additionally reviewed by MR. These were randomly selected but stratified by inclusion/exclusion by IY. Any disagreements were discussed and resolved by ES where necessary. IY and MR initially disagreed on 11 studies (9.43\%). Of these, one was a study identified as eligible by MR, which was initially missed by IY. This was subsequently included in the descriptive synthesis of longitudinal relationships.

Data were extracted (according to the headings in Table 1) from all included studies by IY. Data were also extracted from a randomly selected $49 \%$ of studies $(n=30)$ included in the meta-analysis by PW or AW. IY and the other researchers agreed on 179 out of 208 items entered into the characteristics table (86\%). Where discrepancies in data extraction were identified, these were discussed, and consensus agreed. Of the 29 discrepancies between coders, three were mistakes by the primary coder.

\section{Statistical Analyses}

Whether authors have designated a child or parent factor as the outcome variable, cross-sectional studies cannot provide evidence for relationships over time. Therefore, single timepoint analyses are undifferentiated according to whether the child or parent variable was the nominated outcome.

Two sets of separate meta-analyses were planned. One set included studies reporting effect sizes pertaining to PS (analyses 1-3) and the other to parental MHP (analyses 4-7). Within each set, one analysis was planned for each of: total child EBP (analyses 1 and 4); externalizing problems (analyses 2 and 5); internalizing problems (analyses 3 and 6); total EBP reported by an alternative informant (analysis 7). The alternative informant had to be someone other than the parent who completed the self-report PS or MHP measure, for example, the child's other parent, a teacher or the child themselves. The alternative informant analysis was not run for its association with PS, since only 3 studies provided eligible effect sizes. A final (post-hoc) analysis (analysis 8) was performed for the association between total child EBP and PS measures that did not include a subscale primarily rating aspects of child behavior.

\section{Choice of Effect Size}

In order to ensure independence of data points, only one effect size per study was selected for each meta-analysis. A set of selection rules were established a priori, for cases in which studies provided multiple eligible effect sizes. Firstly, where these corresponded to multiple parental respondents, the effect size for mothers was selected because they represented the majority of respondents in most studies.

In cases where multiple effect sizes arose from use of more than one measure of a concept of interest, more widely-used and better-validated instruments were chosen. Frequency scores were favored over intensity or severity ratings. For entry into the child total problems analyses, a total EBP score was preferred. When this was unavailable, an externalizing (rather than internalizing) subscale score was entered. These were selected because in included studies in which effect sizes for externalizing, internalizing and total score were available, effect sizes based on externalizing scores were better statistical predictors of those for total score ( $\beta=.62$ for externalizing and $\beta=.46$ for internalizing).

For parent measures, a total score on any given PS or MHP instrument was preferred, since these were the most widely reported and tend to be based on more items. In the absence of a total PS score, subscales pertaining to parental perception of own stress levels were preferred over those pertaining to child behavior or other potential sources of stress. For parental MHP, in the absence of a total MHP score, where either an anxiety or a depression scale was available, an anxiety scale was selected. From existing research, it was unclear whether anxiety or depression ratings were more representative of total MHP. However, in our analysis, meta-regression was used to test whether the type of scale used (anxiety, depression or total MHP) statistically moderated effect size.

\section{Calculation of Pooled Effect Sizes}

Statistical analysis was performed with R statistical software version 3.2.4. (R Core Team 2013) using the Metafor package (Viechtbauer 2010). Raw effect sizes were transformed to Fisher's $z$ before synthesis in order to stabilize variance (Borenstein et al. 2009). Along with corresponding sample sizes, these were entered into random effects models in order to estimate pooled effect sizes. These were converted back to Pearson's $r$ scale for interpretation. Random effects models were used, due to the known differences between studies in participant characteristics and measures. This meant that sample statistics from different studies were likely to be estimates of varying population parameters. Heterogeneity across studies was examining using the $Q$ statistic and $I^{2}$. $I^{2}$ values of $\sim 25, \sim 50$, and $\sim 75 \%$ were interpreted as low, moderate, and high, respectively (Higgins et al. 2003). The analyses were then rerun with mean child age, recruitment source and measurement characteristics entered as potential moderators in order to assess their ability to explain between-study variance. Egger et al. (1997) regression test was used to statistically test for funnel plot asymmetry (association between study size and effect size), which may be indicative of publication bias. In the presence of significant 


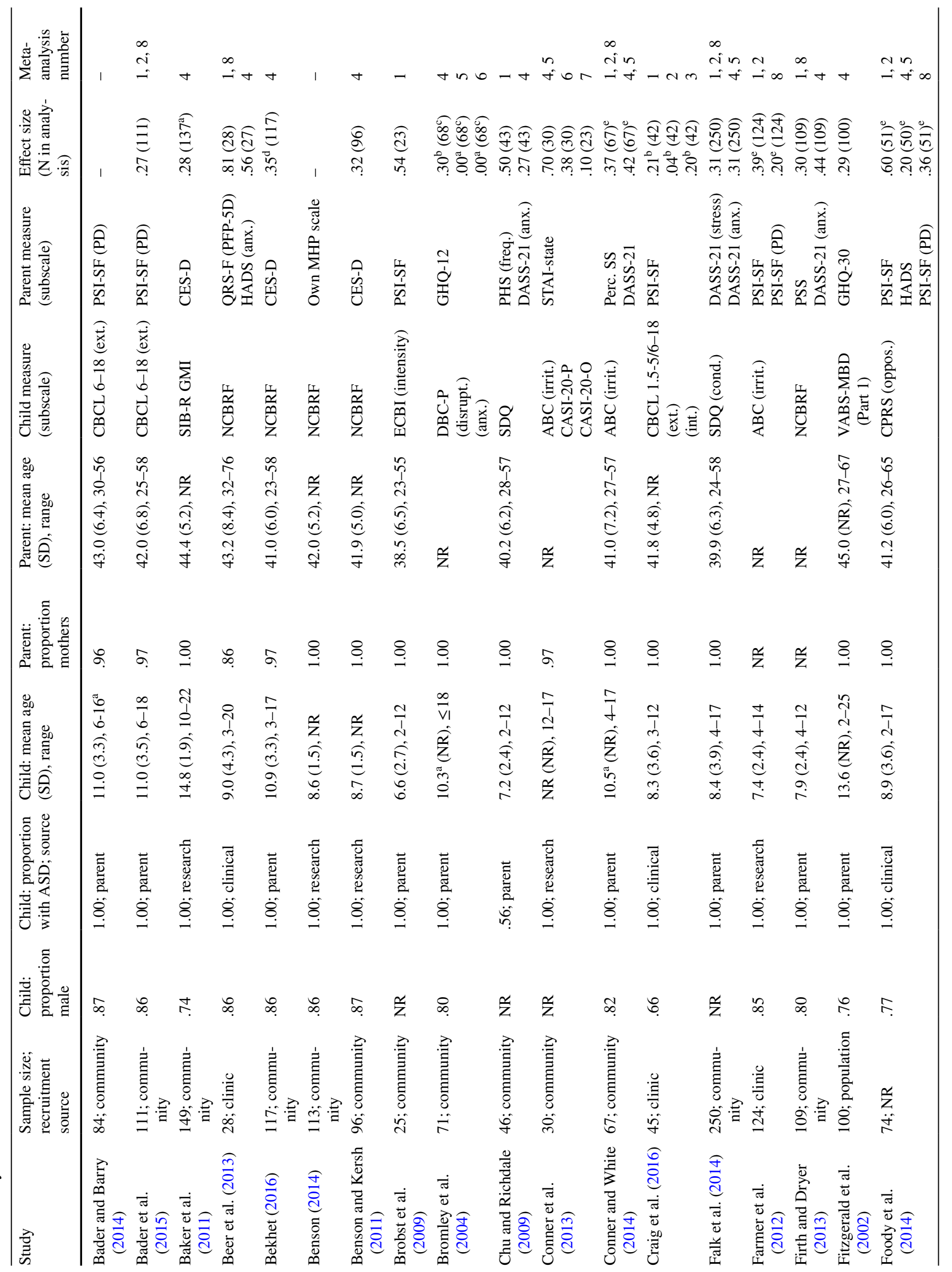




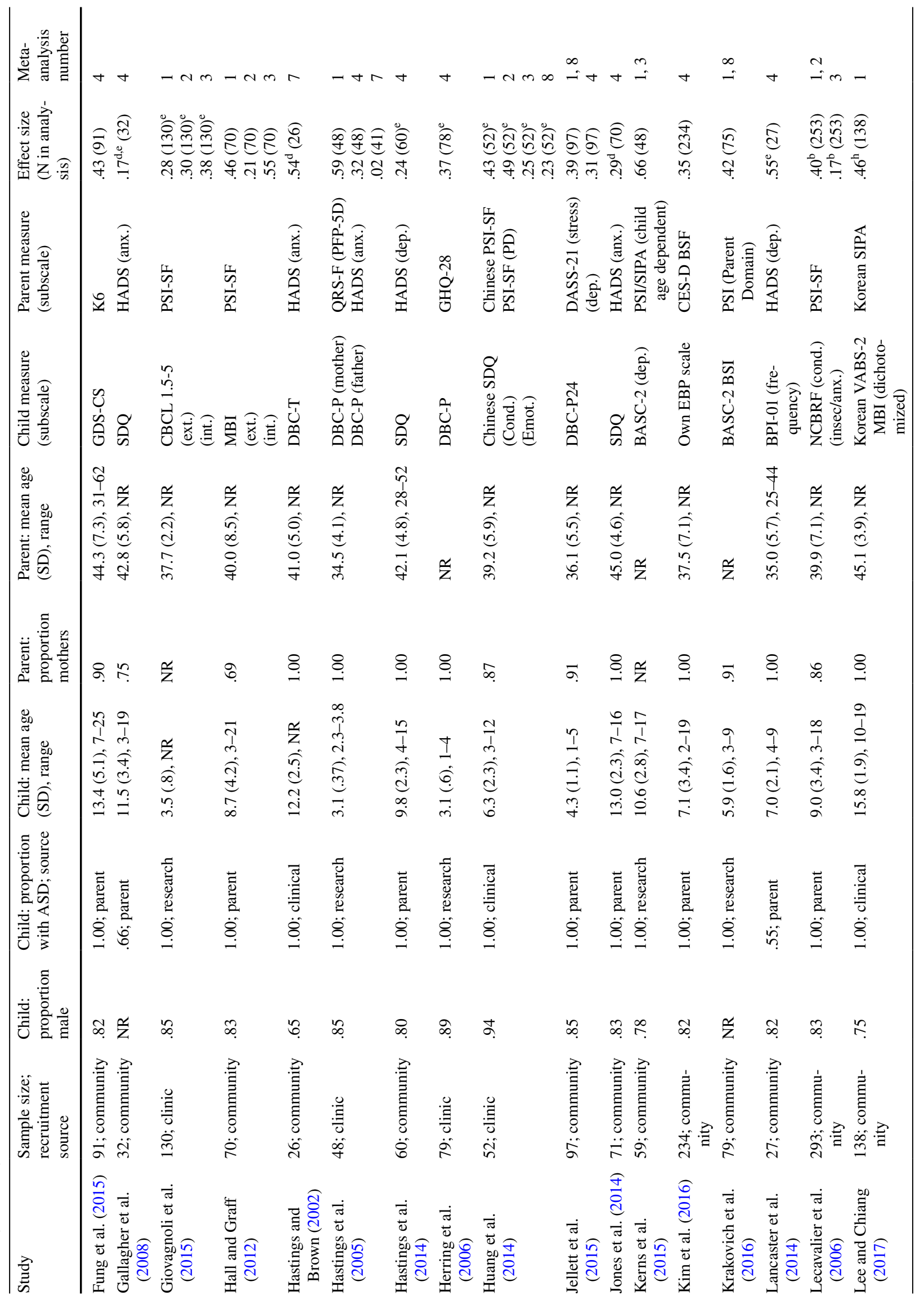




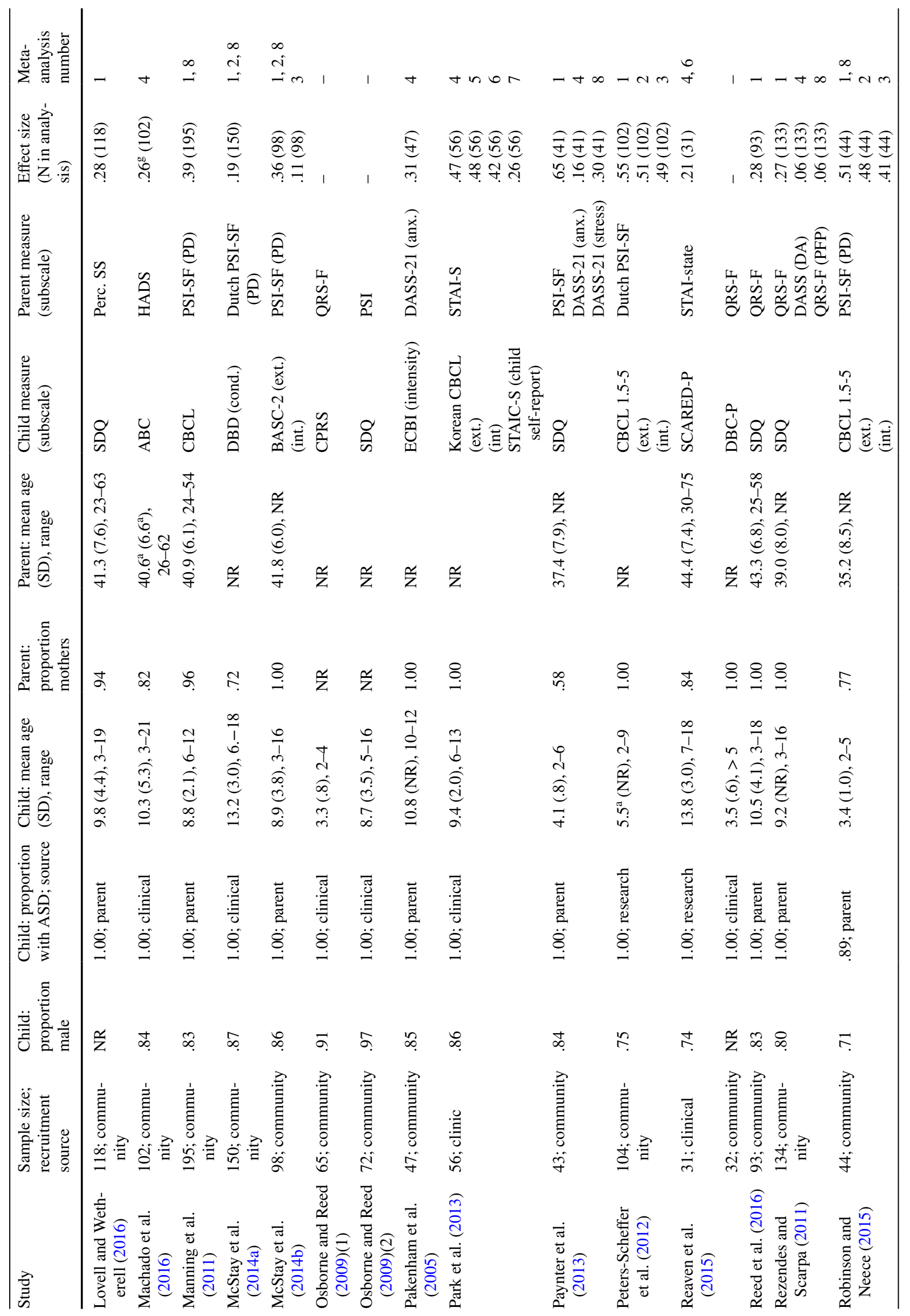




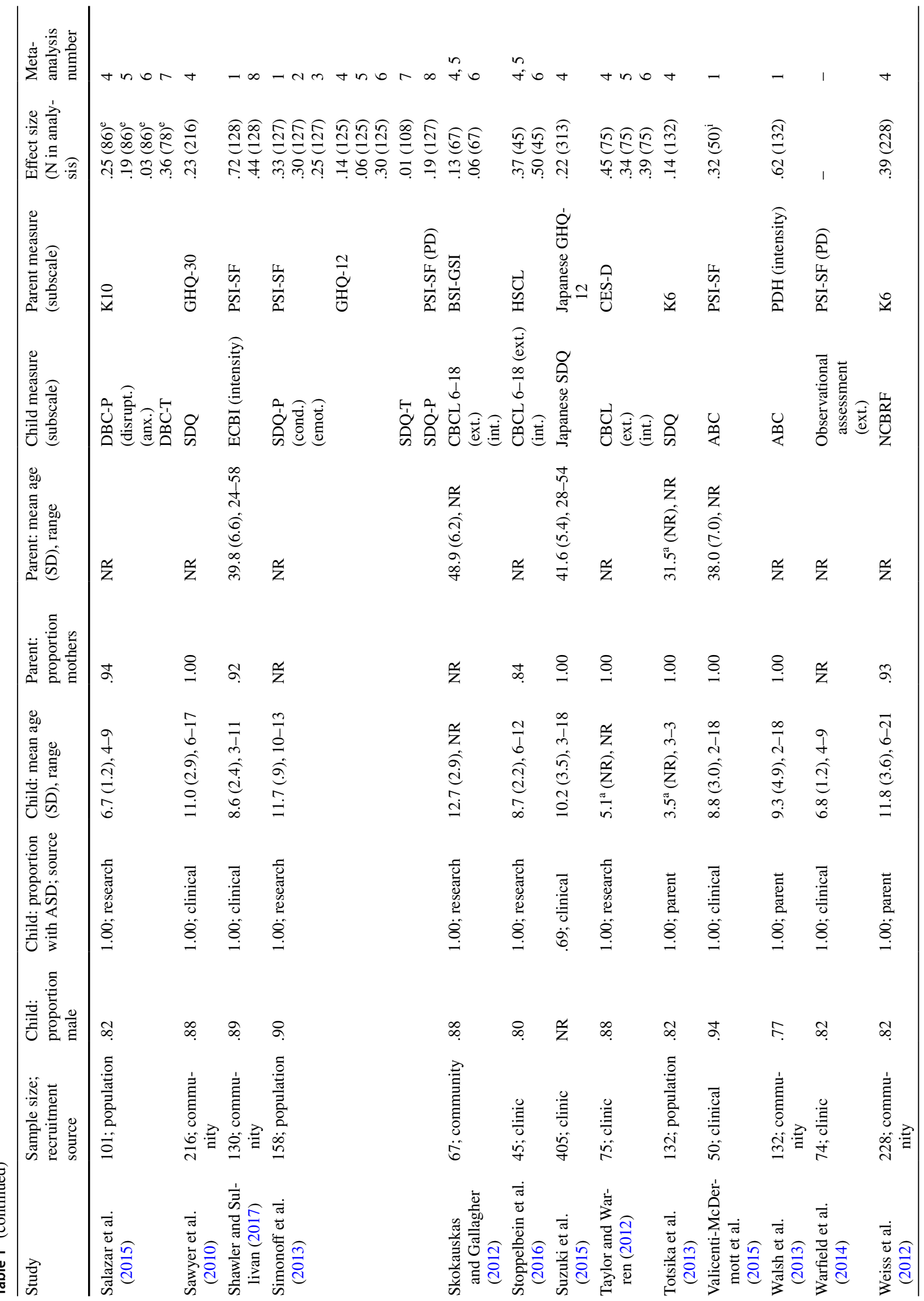




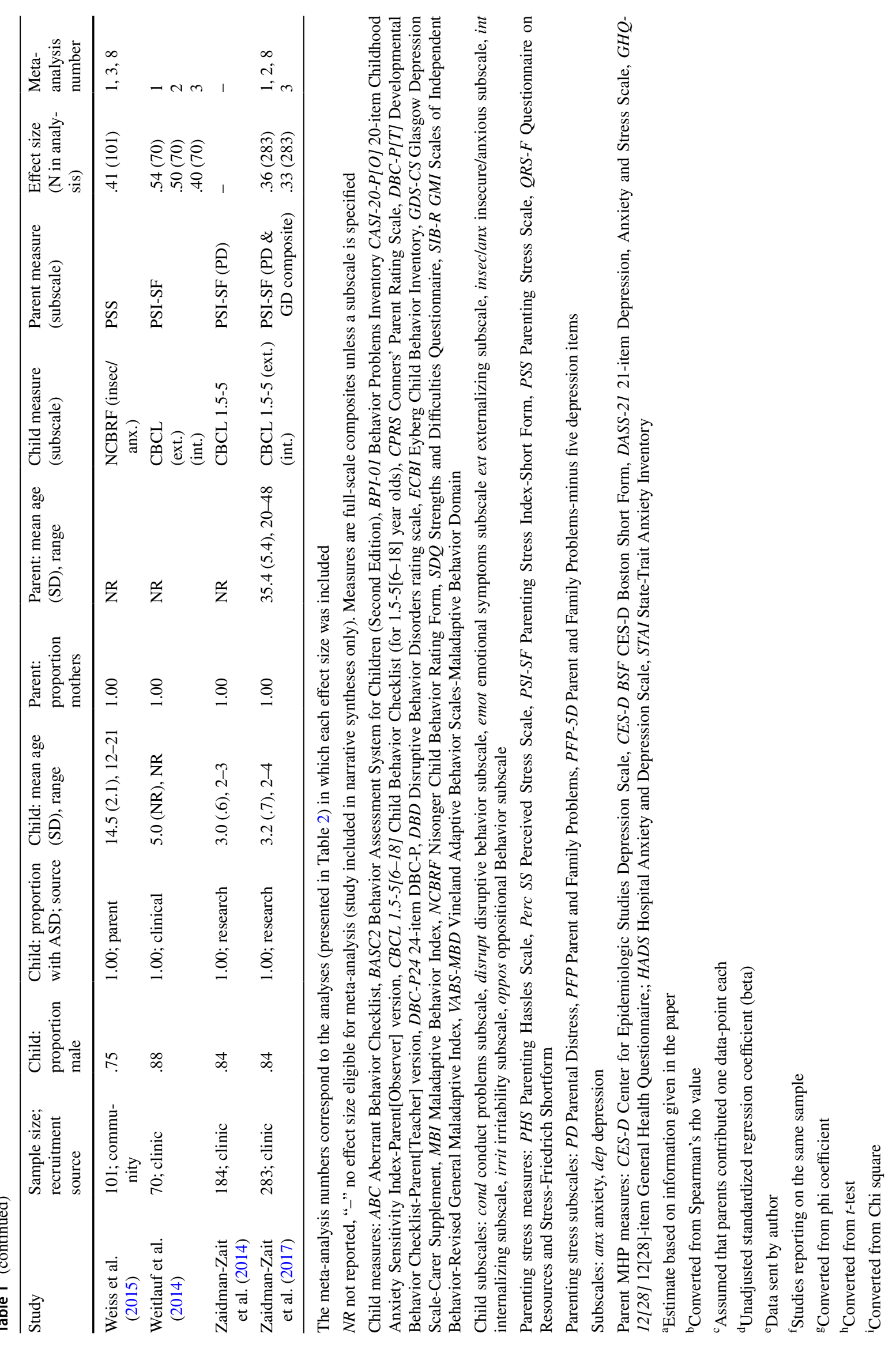


funnel plot asymmetry, we planned to apply the trim and fill procedure (Duval and Tweedie 2000) to correct for upward bias of pooled effect size.

\section{Analysis of Adjusted Concurrent Relationships}

Studies reporting analyses of relationships between the child and parent variables of interest whilst adjusting for other variables were examined in pursuit of Objective 3 . Such analyses included partial and semi-partial correlations between child EBP and either parent MHP or PS, controlling for other variables. Multiple regressions and ANCOVAs with child EBP, parent MHP or PS as the dependent variable (DV) were also relevant. Analyses in which child EBP was the DV were required to include parent MHP or PS as an independent variable (IV) alongside one or more other variables. Analyses in which parent MHP or PS was the DV were required to include child EBP as an independent variable alongside one or more other factors. If child EBP and parent psychological distress share a unique relationship, significant concurrent relationships should be seen despite adjustment for other variables. Other variables appearing in two or more analyses were also assessed for their contribution to the DV, and whether this appeared to be independent of the relationship of interest.

\section{Analysis of Longitudinal Relationships}

Studies that analyzed the relationship between child EBP and parent MHP or PS across time were reviewed descriptively in order to fulfil Objective 4. Eligible analyses included cross time correlations, regressions and Structural Equation Modelling (SEM) techniques. If child additional EBP and parent psychological distress influence each other over time, significant predictive relationships should be seen such that earlier child EBP predicts later (and/or change over time in) parent psychological distress and vice versa.

\section{Results}

\section{Systematic Search}

Figure 1 shows the search and screening process. Electronic database searches identified 5441 papers, of which 3649 remained after removal of duplicate records. After screening by publication type, title and abstract, 414 publications remained. For these studies, full text was retrieved and assessed for eligibility. Sixty-five publications met criteria for review. The most common reasons for exclusion at the full text stage were insufficient sample size and lack of an adequate parent or child measure. An additional two articles were identified and checked for eligibility through manual searching of email alerts and reference lists of eligible studies. Sixty-one included studies were eligible for entry into the meta-analysis after any necessary additional data had been provided by study authors. Study characteristics are reported in Table 1.

\section{Meta-Analyses of Single-Time Point Studies (Objectives 1 and 2)}

Table 2 provides the total number of effect sizes $(k)$, number of independent participants $(N)$ and pooled correlation coefficients for all analyses. For analyses 1-6 (for which the same parent reported on both their own and their child's psychological well-being), these show significant average associations of low to moderate strength $(.25 \leq \bar{r} \leq .43$, CIs [.12-.49]). Within each parent factor, estimates were very similar across analyses for externalizing and internalizing difficulties, and confidence intervals overlapped (PS-externalizing $\bar{r}=.36$, CI [.31-.40]; PS-internalizing $\bar{r}=.36$, CI [.27-.44]; MHP-externalizing $\bar{r}=.29$, CI [.17-.40]; MHPinternalizing $\bar{r}=.25$, CI [.12-.37]). Looking across parent factors, PS showed a significantly stronger pooled association with total child EBP than did parent MHP, as shown by non-overlapping confidence intervals (PS-total EBP $\bar{r}=.43$, CI [.38-.49]; MHP-total EBP $\bar{r}=.30$, CI [.26-.34]).

$Q$ and $I^{2}$ statistics showed most analyses to have significant between-study heterogeneity, in the moderate range. Study heterogeneity was investigated more closely for Analyses 1 and 4, since these provided most power to detect factors which may account for variation between studies. Heterogeneity in the PS-total EBP analysis was particularly high $\left(Q=106.32, p<.001 ; I^{2}=70.73 \%\right)$, whereas the studies forming the MHP-total EBP analysis showed lower but still significant heterogeneity $\left(Q=51.38, p=.03 ; I^{2}=30.64 \%\right)$.

In the PS-total EBP analysis, mean child age was found not to account for variance effect size for either PS or MHP ( $b=-.06, p=.30)$. Recruitment source was also found not to significantly explain variance in effect size $(b=.02 ; p=.72)$. It was noted, however, that several influential studies (of large effect and sample size) had used a Parenting Stress Index (PSI) total score as the measure of PS (Kerns et al. 2015; Paynter et al. 2013; Shawler and Sullivan 2017). This includes the "Difficult Child" subscale, which predominantly comprises ratings of children's behavior problems. The possibility that overlap in content between child EBP measures and PS measures including ratings of child behavior had inflated associations between the two, was therefore investigated.

A binary variable was created denoting whether the measure used included a subscale or full scale in which parents primarily rated child characteristics (1) as opposed to rating their own psychological responses to parenting (0). When entered into a meta-regression, the effect of this factor 
Table 2 Pooled effect sizes

Parenting stress Parent mental health problems

Parenting stressrefined measure criteria

Total difficulties
Meta-analysis number
Pooled $r(95 \% \mathrm{CI})$
$k$
$N$
$Q$
$I^{2}$

1
$.43 * * *(.38-.48)$
35
3625
$106.81 * * *$
$69.77 \%$

Egger's regression test $(z)$

Trim and fill $n$ missing studies

Pooled $r$ after trim and fill

Fail-safe $N$

Externalizing

Meta-analysis number

Pooled $r(95 \%$ CI)

$k$

$N$

$Q$

$I^{2}$

\section{Internalizing}

Meta-analysis number

Pooled $r(95 \% \mathrm{CI})$

$k$

$N$

$Q$

$I^{2}$

Total difficulties-alternative rater

Meta-analysis number
Pooled $r(95 \% \mathrm{CI})$
$k$
$Q$
$N$
$I^{2}$

4

$.30 * * *(.26-.34)$

35

3458

$51.38 *$

$30.64 \%$

$1.72^{\dagger}$

3

$.30 * * *(.25-.33)$

5

$.29 * * *(.17-.40)$

11

919

$28.77 * *$

$68.49 \%$

6

$.25 * * *(.12-.37)$

9

583

$19.14 *$

$59.22 \%$
8

$.34 * * *(.28-.39)$

22

2430

$48.7 * * *$

$54.81 \%$

$2.81 * *$

0

N/A

2206

$k$ number of effect sizes included; $N$ total number of individuals

${ }^{\dagger} p<.10 ; * p<.05 ; * * p<.01 ; * * * p<.001$

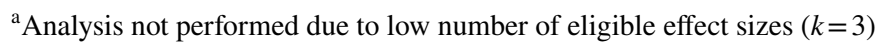

accounted for $13.64 \%$ of heterogeneity between studies, but was not significant $(b=.11 ; p<.09)$. Subset analysis showed a lower average correlation for studies coded as $0(\bar{r}=.38$, CI [.31-.42], $k=15)$ than for those coded as $1(\bar{r}=.47$, CI [.40-.54], $k=20$ ), though confidence intervals overlapped. This suggested that the original pooled estimate for the magnitude of the association between PS and child EBP may have been inflated by the conceptual overlap in some studies. Many studies reporting an effect size for a PS scale including child characteristics, also reported one for a subscale not including them. A further meta-analysis was run in which these were replaced (analysis 8). This analysis showed a somewhat reduced, but still significant, pooled effect size $(\bar{r}=.34$, CI [.28-.39]).

Heterogeneity in the MHP-total EBP analysis was not explained by child age $(b=.01, p=.90)$. We also tested whether the scale or subscale of the parent MHP measure used could explain heterogeneity. Whether studies used a total score, an anxiety subscale or a depression subscale did not appear to be related to effect size $(b=.01, p=.84)$. Recruitment source (clinic, community or population) was not a significant explainer of heterogeneity $(b=.07 ; p=.08)$. 
Study identification

Screening Process

Exclusions

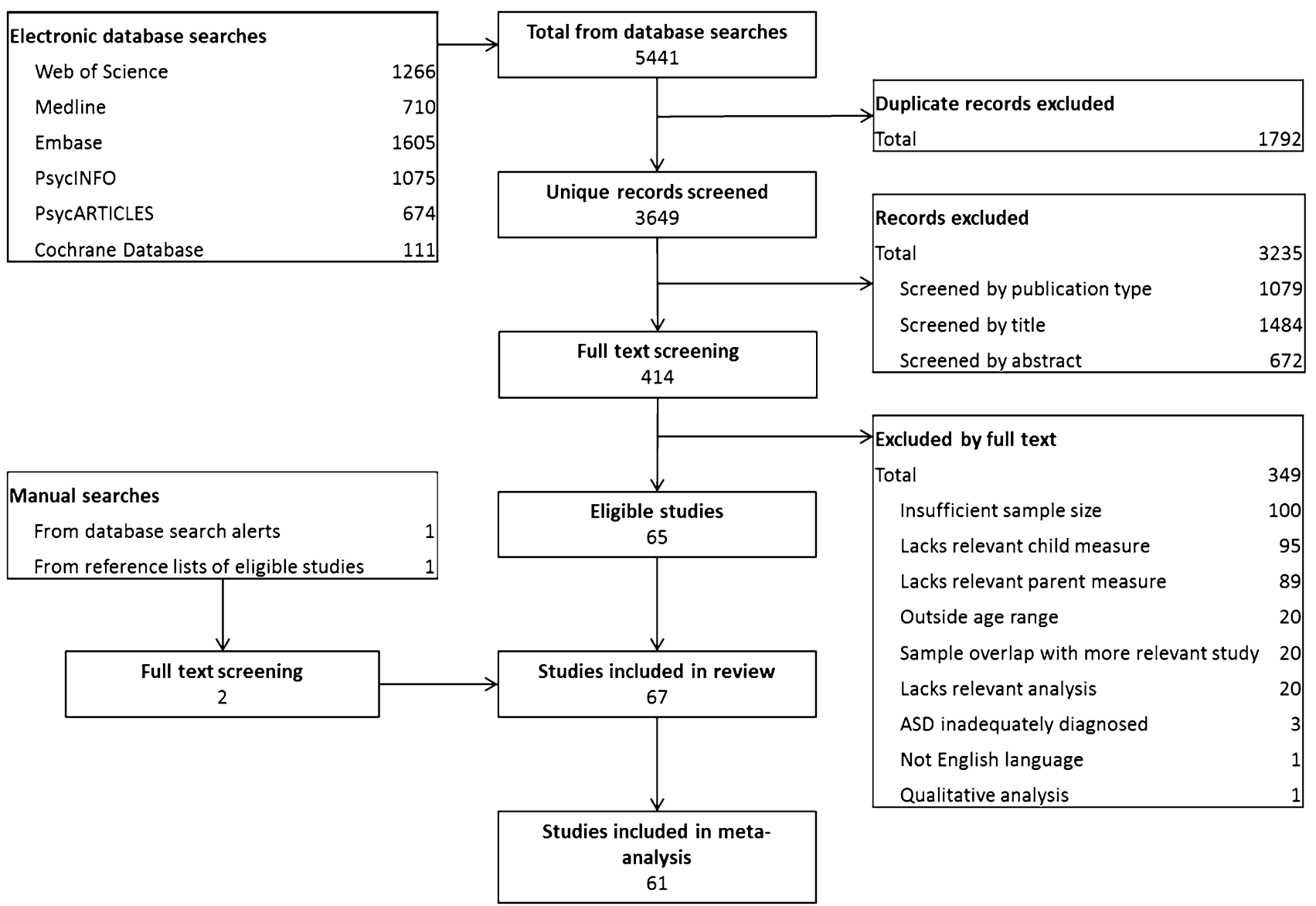

Fig. 1 Search results and screening process

Subset analyses revealed that pooled $r$ for populationderived samples was .20 (CIs [.11-.29]; $k=4$ ). For community samples $\bar{r}=.31$ (CIs [.27-.36]; $k=25$ ) and for clinical samples $\bar{r}=.36$ (CIs [.23-.47]; $k=6$ ).

Among studies measuring parent MHP, six provided an effect size of association with child EBP rated by someone other than the parent reporting own MHP. These were entered into a separate meta-analysis (analysis 7), which showed a lower, but still significant pooled magnitude of association ( $\bar{r}=.21$, CI [.04-.37]). Heterogeneity for this analysis was moderate $\left(I^{2}=55.92 \%\right)$, which may reflect the range of alternative raters providing data (e.g. another parent, teacher, child self-rating). However, this could not be tested given the small number of studies. For PS, only three studies provided alternative rater EBP scores, which was deemed insufficient to conduct a meaningful meta-analysis.

Potential publication bias was assessed in the PS-total EBP and MHP-total EBP analyses. For the MHP-total EBP analysis, Egger's regression test showed no significant evidence for funnel plot asymmetry $(z=1.72, p=.08)$.
The trim and fill procedure imputed 3 "missing" studies; however, the pooled estimate remained largely unchanged, (see Table 2). Assessing publication bias in the total EBPPS analysis was problematic, since conventional tests are unsuitable in the presence of substantial heterogeneity (Peters et al. 2010). For this reason, publication bias was assessed in analysis 8 , in which heterogeneity was somewhat reduced. Here, there was significant evidence for funnel plot asymmetry $(z=2.81, p<.001)$. However, performing the trim and fill procedure did not impute any "missing" studies, possibly due to the still-considerable heterogeneity, even among large studies. Instead, we assessed tolerance for unpublished null results using Rosenthal's (1979) fail-safe $N$ procedure. This showed that an additional 2206 non-significant effect sizes would be required to reduce the pooled association such that the $p$-value reaches the .05 threshold. 


\section{Adjusted Single Time-Point Associations (Objective} 3)

Thirty-one studies provided data relevant to Objective 3 . Across studies, numerous additional parent, child and contextual variables were included in multivariate analyses alongside the IV of interest, potentially sharing its relationship with the DV. In order to synthesize findings, analyses were split into those relevant to the PS-EBP relationship and those relevant to the MHP-EBP relationship. The impact of additional variables that were included in two or more analyses in either set was examined. It was noted firstly, whether the relationship of interest remained significant whilst adjusting for other variables. Secondly, it was noted which variables made a significant independent contribution to the DV. This information is presented for the PS-EBP relationship in Table 3 and for the MHP-EBP relationship in Table 4. Common additional variables included child ASD severity, family financial situation (income or SES), social support, family functioning and parents' perception of their own parenting (e.g. parenting self-efficacy or endorsement of positive/negative parenting practices). In the majority of analyses, the relationships of interest remained significant, despite accounting for additional child, parent and contextual variables. However, this was not universally true. Important additional factors are now discussed with reference to their ability to statistically account for the relationships of interest and their independent contributions to variance in the DV.

\section{Child Factors}

Child age, sex, IQ and adaptive behavior very rarely showed association with the DV. Where they did, the relationship of interest (EBP-PS or EBP-MHP) was also independently significant. Therefore, these child factors did not appear to account for the associations of interest to any important extent. Several studies report the PS-EBP relationship to remain significant despite accounting for ASD severity, which itself was not related to the dependent variable (Giovagnoli et al. 2015; Hastings et al. 2005; Manning et al. 2011; McStay et al. 2014a; Weiss et al. 2015; Zaidman-Zait et al. 2017). However, three studies found no significant PS-EBP relationship whilst accounting for ASD severity (Bader et al. 2015; Falk et al. 2014; Firth and Dryer 2013). In these studies, child ASD severity did significantly account for variance in the DV. It therefore remains a possibility that the relationship between PS and EBP is to some extent accounted for by ASD severity. Regarding parent MHP, three studies found a significant relationship with EBP whilst accounting for ASD severity (which itself did not contribute to explained variance). Only Falk et al. (2014) report a significant association between MHP and ASD symptoms, whilst child EBP did not reach the criterion for entry into this stepwise regression.

\section{Parent Factors}

Parent age was entered in five studies, but was only significantly related to the dependent variable in that of Falk et al. (2014). Here, increasing maternal age was related to lower levels of maternal stress $(\beta=-.24, p<.001)$ and anxiety $(\beta=-.23, p<.001)$, whilst child EBP did not share unique variance with either of these dependent variables. This was a well-powered study, thus parental age cannot be ignored as a potential statistical mediator of the relationships of interest.

Parent use of coping mechanisms was investigated in three studies with PS as the DV (Manning et al. 2011; McStay et al. 2014b; Zaidman-Zait et al. 2017). Although coping mechanisms showed significant relationships with PS, child EBP retained a significant association, suggesting it has an independent effect. On the other hand, when parent perception of their own parenting was present in the models, the relationship of PS and parent MHP with child EBP became non-significant in three (Bader et al. 2015; Falk et al. 2014; Hastings and Brown 2002) out of four studies. Only in the largest study did both child EBP and parent perception of their own parenting both appear to contribute significantly to parent MHP (Suzuki et al. 2015). Parents' perception of their ability to parent effectively is therefore a plausible candidate for a mediator of the relationship between child EBP and parent psychological distress variables.

\section{Family and Contextual Factors}

Perceived social and family support was commonly included in analyses and often made a significant contribution to variance in the DV. However, in most cases, the relationship of interest also remained significant. Cases in which the relationship did not remain significant tended to have a smaller sample size (e.g. Warfield et al. 2014; Gallagher et al. 2008). This suggests that social and family support may have an important relationship with parent MHP and PS; however, this relationship is independent of that with child EBP. Socio-economic status and family income rarely showed significant association with the dependent variables. Although some degree of relatedness to child EBP (Weiss et al. 2015), PS and parent MHP (Jones et al. 2014) was observed, the relationships between parent and child variables of interest remained significant in these cases.

\section{Longitudinal Analyses (Objective 4)}

Baseline characteristics of the eleven included studies are provided in Table 1 and data regarding longitudinal analyses are given in Table 5. A variety of design and statistical 


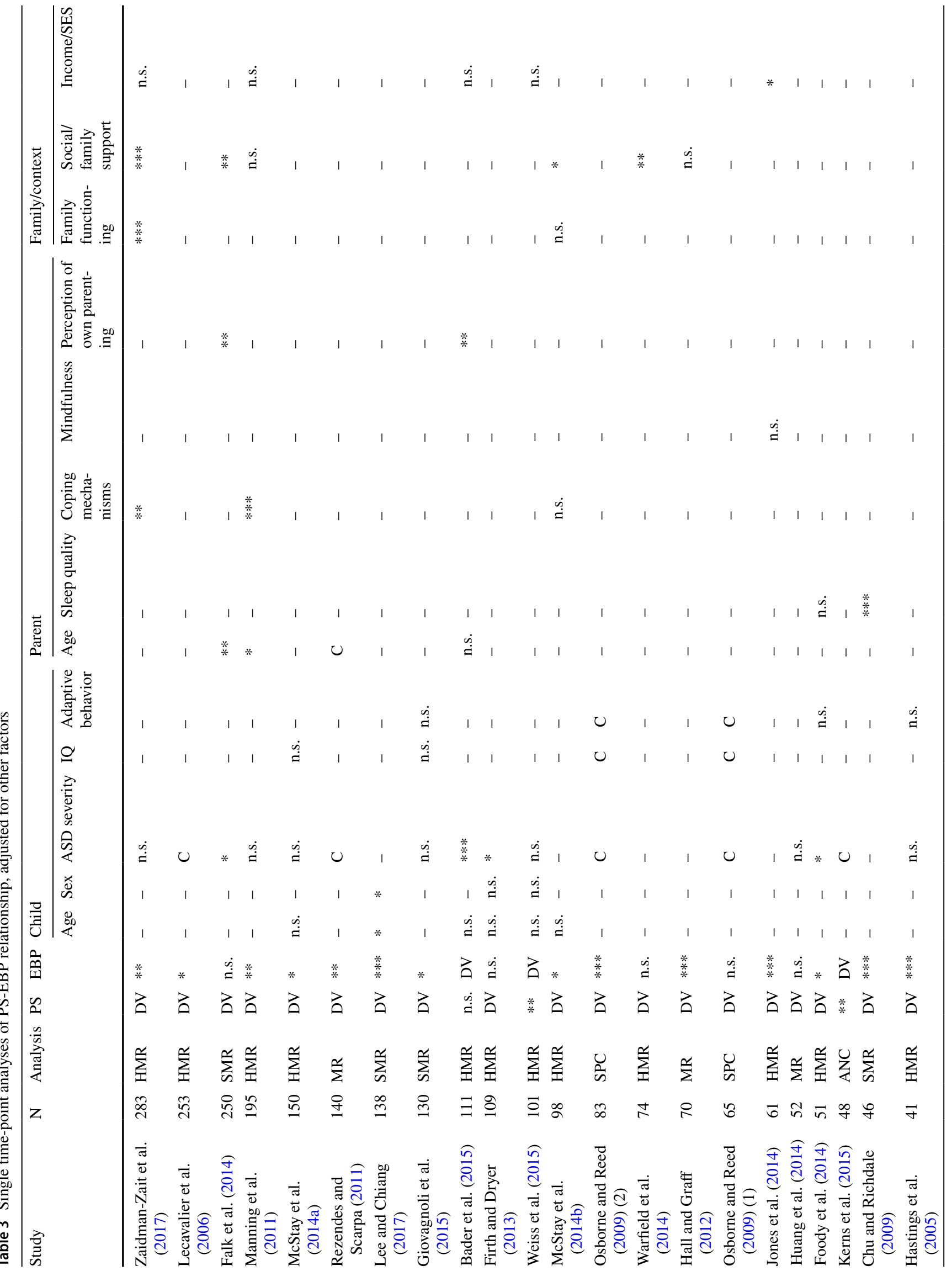


approaches has been taken among these studies. Cross-time zero-order correlations (unadjusted association between a measure at an earlier and another measure at a later timepoint) showed significant relationships between earlier PS and later child EBP in two studies of over 100 participants (Peters-Scheffer et al. 2012; Zaidman-Zait et al. 2014), but not in three studies with under 100 participants (Bader and Barry 2014; Reed et al. 2013; Simonoff et al. 2013). A similar pattern was shown for the association of earlier child EBP to later PS, and both directions of association between child EBP and parent MHP (Baker et al. 2011; Simonoff et al. 2013; Totsika et al. 2013).

However, in analyses controlling for variable stability over time (i.e. the association of a DV with itself at an earlier time-point), associations tended to disappear (Baker et al. 2011; Hastings et al. 2014; Peters-Scheffer et al. 2012). Exceptions included the largest study (Zaidman-Zait et al. 2014), which found that earlier child EBP predicted later PS and vice versa (though only in the second time interval). In a smaller study, Lecavalier and colleagues (2006) found similar bidirectional associations, though the effect size was larger for the parent-to-child than the child-to-parent. Another large study (Totsika et al. 2013) found earlier parent MHP to predict later child EBP, but not vice versa.

Significant cross-time relationships have also been found whilst adjusting for other factors. Osborne and Reed (2009) found in two samples that earlier PS predicted later child EBP, whilst controlling for child characteristics (ASD severity, cognitive ability and adaptive functioning). The opposite (child-to-parent) predictive relationship was not evident. It may be that earlier child characteristics share their predictive relationship for PS, whereas earlier PS shows predictive ability for EBP that is separate from other child characteristics such as ASD severity.

Finally, Benson (2014) investigated moderation of the association between child EBP and change over time in parent MHP by parent use of a variety of coping mechanisms. He found an attenuated EBP-MHP relationship in parents who reported high use of a cognitive reframing strategy. This strategy involves actively changing ones perception of a stressor. Despite inclusion of this interaction term in the multilevel modelling analysis, the relationship between child EBP and change in parent MHP remained significant.

\section{Discussion}

This review aimed systematically to collate the published evidence pertaining to the relationships between additional EBP in children with ASD and their parents' MHP and PS. A series of meta-analyses showed the magnitude of unadjusted associations between these factors to be in the moderate range. The pooled estimates for the MHP-EBP analyses 


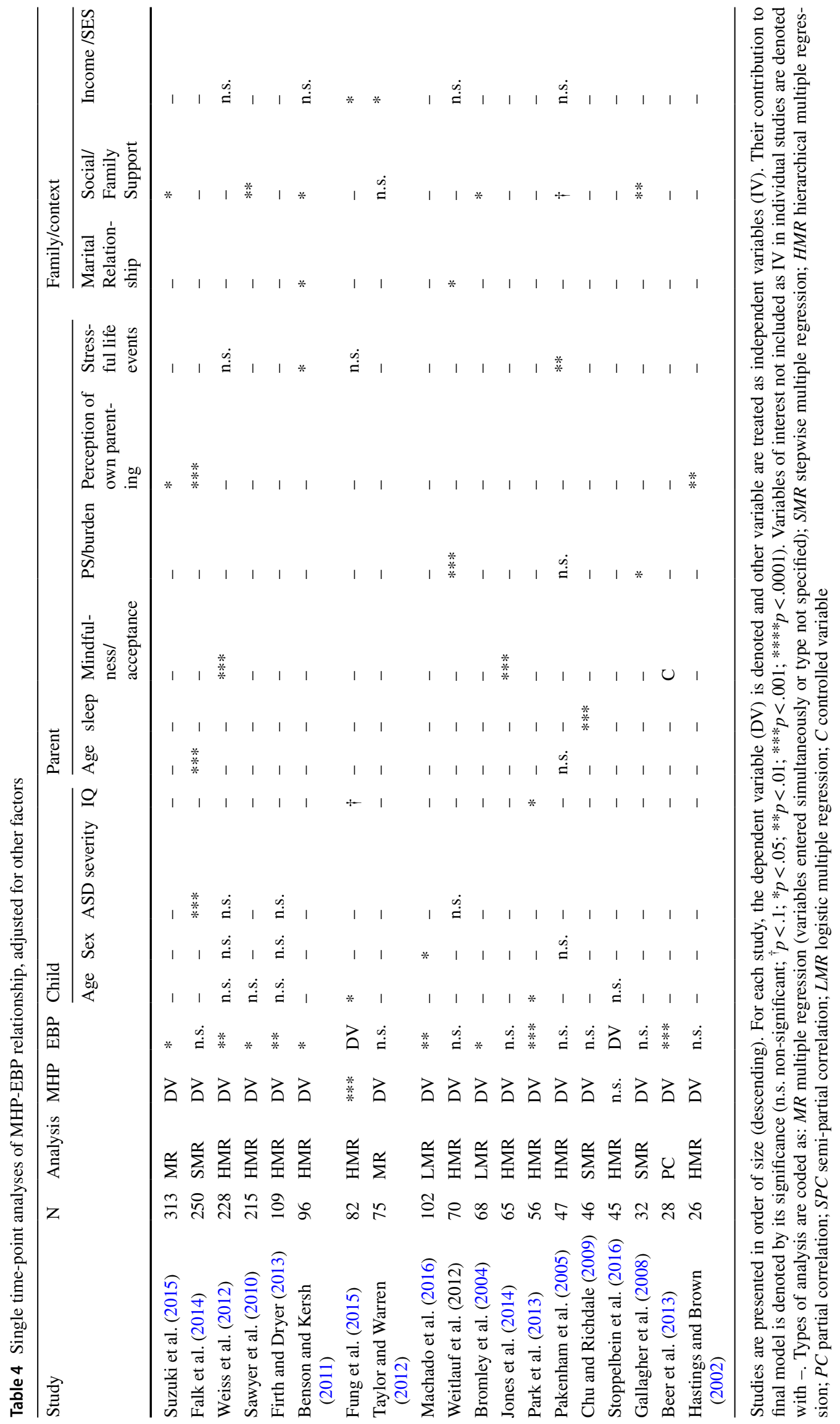




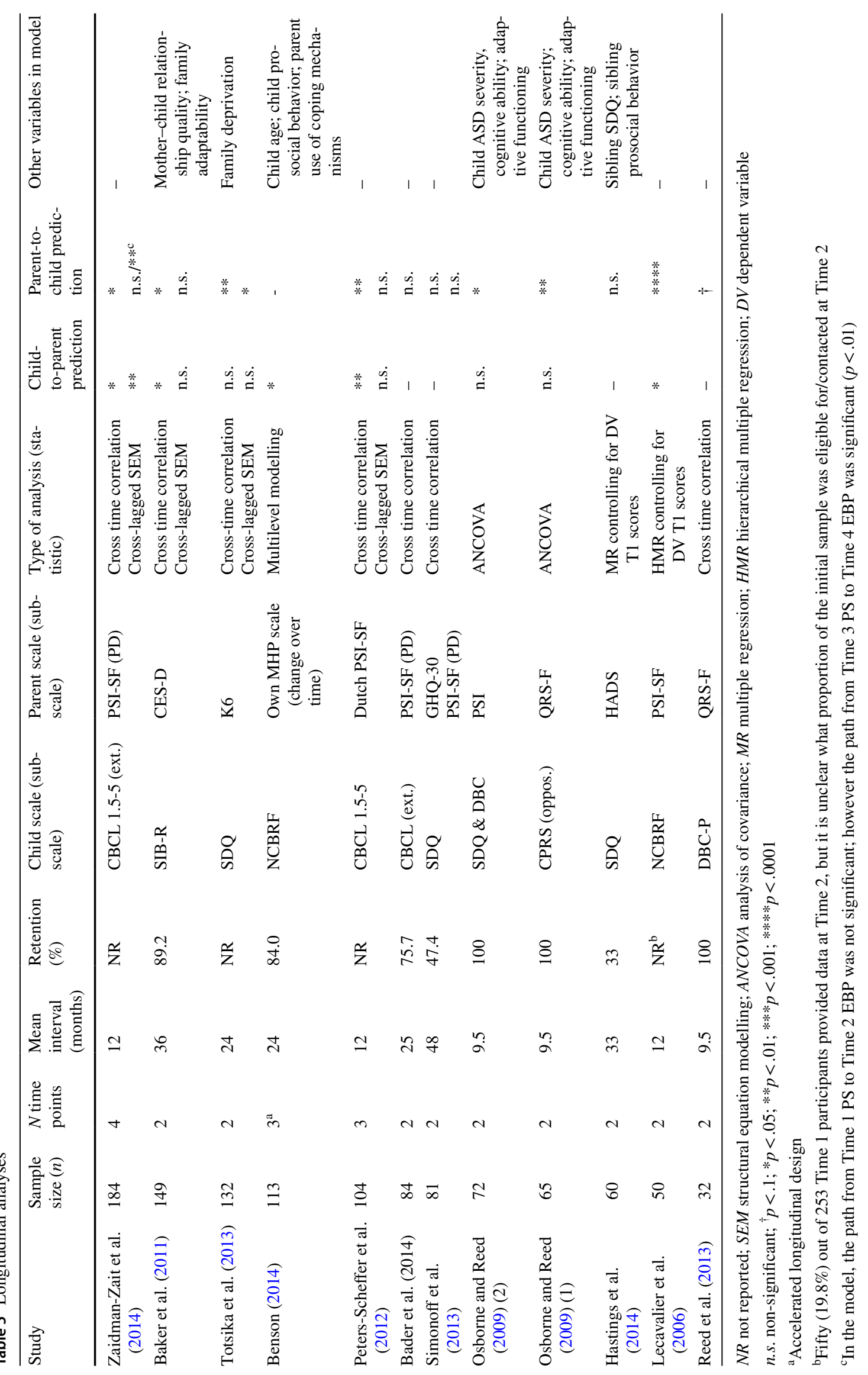


were slightly stronger than those found in comparable metaanalyses conducted by Goodman et al. (2011) in the general population. This remained true after controlling for inflation by small study effects (using the trim and fill procedure). Pooled correlation was lower in our analysis of effect sizes based on measures of child EBP rated by an alternative informant to the parent self-reporting on own MHP. However, when Goodman and colleagues performed comparable subset analyses, pooled estimates remained lower than our corresponding results for child EBP ratings by the mother, child themselves and by an observer. Goodman et al. did find a stronger pooled correlation $(\bar{r}=.34)$ for studies in which mothers were recruited from clinical settings. Since mothers of children with ASD are already at increased risk for clinically relevant psychological distress (Jokiranta et al. 2013), our results and those of Goodman et al. appear to provide converging evidence that at higher levels of parental psychological distress, its relationship with child EBP is stronger. Although the current research was not equipped for further investigation of this possibility, our findings highlight the importance of investigating these associations in families of children with ASD. Consistent with Goodman and colleagues' research, associations appeared similar for internalizing and externalizing child difficulties. Indicating the need to continue to investigate both of these factors in future research.

The PS-total EBP pooled association appeared greater in magnitude than that for MHP-total EBP; however, it also showed higher heterogeneity. We found some evidence that this heterogeneity may be driven at least in part by the similarity in content between child EBP questionnaires, and PS questionnaires that include ratings of child behavior. Several research groups have chosen to use subscales of PS questionnaires that minimize overlap between the concepts of child behavior problems themselves, and parent perception of the stress related to them (Bader et al. 2015; Simonoff et al. 2013; Zaidman-Zait et al. 2014). This approach may be advantageous in future research. Regarding MHP measures, there was no evidence that type of parent MHP measured (depression, anxiety or both) accounted for variation in effect size reported by different studies. It is worth acknowledging that parental MHP is not limited to mood disorders such as depression and anxiety and that other classes of mental health disorder (e.g. psychosis) could have different relationships with additional child EBP. However, these remain largely unexplored to date.

In the vast majority of studies, the informant for child EBP was the same parent that rated their own stress and MHP. This is potentially problematic in two ways. Firstly, correlations could be inflated by common method effects (Podsakoff et al. 2003), whereby questionnaire data collected from the same source may be related for reasons other than a true association between the concepts of interest. In this context specifically, it is possible that parents who experience more psychological distress also perceive the same child behaviors as more problematic than do other parents (Najman et al. 2000). Secondly, reliance on a single information source (mainly mothers) for child EBP may limit the applicability of the relationships seen with parent psychological distress to certain conditions. Elements of child behavior that parents find problematic may not align completely with elements that are problematic for others, including the children themselves. However, parent agreement with other informants appears to be moderate for the externalizing and internalizing behavior problems of children with ASD (Stratis and Lecavalier 2015). This suggests that parent-report captures at least some variance in common with that indexed by other reporters.

We found that in six studies where an effect size deriving from an alternative rater was available, the pooled estimate for the EBP-MHP association was lower, but remained significant. These results are similar to those found in the general population by Goodman et al. (2011), in which child EBP ratings by mothers yielded a larger pooled association with maternal depression than those yielded by observer or child self-report ratings (which were nevertheless significant). Together, these findings suggest that although the possibility of inflation by shared rater effects remains, these cannot account for the entire observed association. Using multiple informants may be the best way to home in on a more pervasive element of variance in child EBP (Stratis and Lecavalier 2015).

Our narrative review of adjusted concurrent relationships showed that in general, the associations between child EBP and parent MHP and PS are robust, as they tend to remain present after statistically accounting for other factors. This is especially true in larger studies. However, there is some evidence that this covariance may be shared with ASD severity and parent self-rated psychological factors such as coping mechanisms and perception of own parenting ability. This is supported by longitudinal research finding that the relationship between earlier parenting stress and later child EBP was mediated by parent self-rated ability to set limits for their child's behavior (Osborne et al. 2008). (This study was not included in this review since it was based on the same sample as another study that used analyses more relevant to our aims.) This is consistent with a model proposed by Hastings (2002) in which child EBP increases parenting stress, which in turn compromises a parent's ability to engage in positive parenting behaviors that would usually serve to support the child's emotional and behavioral functioning. It is plausible that parent use of coping strategies to deal with stress may moderate the relationship between child EBP and PS, or between PS and parenting behavior. Evidence for the former was found by Benson (2014), as outlined in the longitudinal analyses section, above. 
Although there was insufficient consistency across studies in other included variables to synthesize evidence regarding mediation and moderation, longitudinal studies provided some consistent evidence for the existence of bidirectional relationships between parent psychological well-being and child EBP over time. Studies with sample sizes of under 100 may be underpowered to detect associations, particularly whilst adjusting for other factors. Research finding bidirectional relationships in the general population has benefited from much larger samples (e.g. Bagner et al. 2013; Stone et al. 2016; Zadeh et al. 2010). This review therefore highlights the value of conducting further well-powered longitudinal research into the temporal relationship between child and parent psychological well-being in families with ASD. Establishing the structure of these relationships, and the additional factors that mediate and moderate them, may yield explanations as to the increased rate of MHP in both children with ASD and their parents, as well as guiding intervention research.

\section{Strengths}

To our knowledge, this is the first systematic review of the growing literature on the relationships between additional EBP in children with ASD and psychological distress in their parents. It provides both a quantitative and qualitative synthesis of such research, yielding a comprehensive overview and basis for guiding future research. Strengths of the meta-analytic component include efforts to obtain additional data from study authors, and reliability checks for the screening and coding of studies. Disaggregation of child externalizing and internalizing problems allowed us to show that the relationships with parent MHP and PS observed for total EBP are likely not solely driven by either, but rather that both may be important factors. This is consistent with research in the general population (Goodman et al. 2011). Strengths of the qualitative syntheses include their focus on specific research questions aiming to guide future research.

\section{Limitations}

A limitation of this review is its reliance on published research. Although there was little statistical evidence for publication bias in the parent MHP-total EBP analysis, significant evidence was present in the PS-total EBP analysis. Unfortunately, it was not possible to adequately disentangle the effects of potential publication bias from residual between-study heterogeneity. However, the number of unpublished null results required to render the PS-total EBP relationship non-significant was very large. Therefore, whilst it remains a possibility that the pooled effect size is inflated due to publication bias, it is unlikely that publication bias can completely explain the observed association.
A further limitation of this review is that it did not consider measurement of PS and MHP, other than by parent self-report. Several recent studies have used cortisol as a biomarker for stress in parents of children with ASD (e.g. Bitsika et al. 2017; Dykens and Lambert 2013). Although not reviewed here, these studies contribute importantly to the literature on PS in ASD, since stress by definition includes a physiological component related to both physical and mental health outcomes (Dykens and Lambert 2013).

\section{Conclusions}

Systematic synthesis of research into the relationship between additional MHP in children with ASD and stress levels and mental health of their parents has overall shown robust relationships both concurrently and across time. Future research should focus on further investigating the additional factors involved in these relationships in an organized and hypothesis-driven manner, based on evidence from existing research. Progression in this essential area of research should come from well-powered longitudinal studies using well-defined measures from a variety of information sources.

Acknowledgments The authors would like to thank Dr. Joanne Tarver for her advice related to this project.

Author Contributions IY ran the search procedures, study screening, data extraction and analysis, and wrote the manuscript. PW, AW and MR contributed to reliability checks on study inclusion and data extraction. Disagreements in study inclusion and data extraction were resolved by ES. TC and ES oversaw the conception and procedures of the study. ES, TC and PW were involved in drafting the manuscript. All authors read and approved the final manuscript.

Funding IY was supported by a PhD studentship funded jointly by Autistica (PCFTAAR) and The Sackler Institute. All authors were either researchers or students working on a National Institute for Health Research (NIHR) funded research program, IAMHealth (RPPG-1211-20016). ES additionally receives support from the National Institute of Health Research (NIHR) Biomedical Research Centre at South London and Maudsley Foundation Trust (IS-BRC-1215-20018), the NIHR through a Senior Investigator Award (NF-SI-0514-10073), the European Union Innovative Medicines Initiative (EU-IMI 115300), Autistica (7237), Medical Research Council (MR/R000832/1, MR/ P019293/1), the Economic and Social Research Council (ESRC 003041/1), Guy's and St Thomas' Charitable Foundation (GSTT EF1150502) and the Maudsley Charity. TC receives grant or research support from the National Institute of Health Research, the Medical Research Council and the European Union. He has received royalties from Guilford Press and Sage Publications.

\section{Compliance with Ethical Standards}

Conflict of interest The authors declare that they have no conflict of interest. 
Research Involving Human and Animal Participants This article does not contain any studies with human participants or animals performed by any of the authors.

Open Access This article is distributed under the terms of the Creative Commons Attribution 4.0 International License (http://creativeco mmons.org/licenses/by/4.0/), which permits unrestricted use, distribution, and reproduction in any medium, provided you give appropriate credit to the original author(s) and the source, provide a link to the Creative Commons license, and indicate if changes were made.

\section{References}

American Psychiatric Association. (2013). Diagnostic and statistical manual of mental disorders (5th ed.). Arlington, VA: American Psychiatric Publishing.

Bader, S. H., \& Barry, T. D. (2014). A longitudinal examination of the relation between parental expressed emotion and externalizing behaviors in children and adolescents with autism spectrum disorder. Journal of Autism and Developmental Disorders, 44(11), 2820-2831. https://doi.org/10.1007/s10803-014-2142-6.

Bader, S. H., Barry, T. D., \& Hann, J. A. H. (2015). The relation between parental expressed emotion and externalizing behaviors in children and adolescents with an autism spectrum disorder. Focus on Autism and Other Developmental Disabilities, 30(1), 23-34.

Bagner, D. M., Pettit, J. W., Lewinsohn, P. M., Seeley, J. R., \& Jaccard, J. (2013). Disentangling the temporal relationship between parental depressive symptoms and early child behavior problems: A transactional framework. Journal of Clinical Child and Adolescent Psychology, 42(1), 78-90.

Baker, J. K., Seltzer, M. M., \& Greenberg, J. S. (2011). Longitudinal effects of adaptability on behavior problems and maternal depression in families of adolescents with autism. Journal of Family Psychology, 25(4), 601-609.

Beer, M., Ward, L., \& Moar, K. (2013). The relationship between mindful parenting and distress in parents of children with an autism spectrum disorder. Mindfulness, 4(2), 102-112.

Bekhet, A. K. (2016). The mediating effects of positive cognitions on autism caregivers' depression and their children's challenging behaviors. Archives of Psychiatric Nursing, 30(1), 13-18.

Benson, P. R. (2014). Coping and psychological adjustment among mothers of children with ASD: An accelerated longitudinal study. Journal of Autism and Developmental Disorders, 44(8), 1793-1807.

Benson, P. R., \& Kersh, J. (2011). Marital quality and psychological adjustment among mothers of children with ASD: Crosssectional and longitudinal relationships. Journal of Autism and Developmental Disorders, 41(12), 1675-1685.

Bitsika, V., Sharpley, C. F., Andronicos, N. M., \& Agnew, L. L. (2017). What worries parents of a child with autism? Evidence from a biomarker for chronic stress. Research in Developmental Disabilities, 62, 209-217.

Bolton, P. F., Pickles, A., Murphy, M., \& Rutter, M. (1998). Autism, affective and other psychiatric disorders: patterns of familial aggregation. Psychological Medicine, 28(2), 385-395.

Borenstein, M., Hedges, L. V., Higgins, J. P. T., \& Rothstein, H. R. (2009). Effect sizes based on correlations. Introduction to metaanalysis (pp. 41-43). Chichester: Wiley.

Brobst, J. B., Clopton, J. R., \& Hendrick, S. S. (2009). Parenting children with autism spectrum disorders the couple's relationship. Focus on Autism and Other Developmental Disabilities, 24(1), $38-49$.
Bromley, J., Hare, D. J., Davison, K., \& Emerson, E. (2004). Mothers supporting children with autistic spectrum disorders-Social support, mental health status and satisfaction with services. Autism, 8(4), 409-423.

Cadman, T., Eklund, H., Howley, D., Hayward, H., Clarke, H., Findon, J., et al. (2012). Caregiver burden as people with autism spectrum disorder and attention-deficit/hyperactivity disorder transition into adolescence and adulthood in the United Kingdom. Journal of the American Academy of Child and Adolescent Psychiatry, 51(9), 879-888.

Chu, J., \& Richdale, A. L. (2009). Sleep quality and psychological wellbeing in mothers of children with developmental disabilities. Research in Developmental Disabilities, 30(6), 1512-1522.

Conner, C. M., Maddox, B. B., \& White, S. W. (2013). Parents' state and trait anxiety: Relationships with anxiety severity and treatment response in adolescents with autism spectrum disorders. Journal of Autism and Developmental Disorders, 43(8), 1811-1818.

Conner, C. M., \& White, S. W. (2014). Stress in mothers of children with autism: Trait mindfulness as a protective factor. Research in Autism Spectrum Disorders, 8(6), 617-624. https://doi. org/10.1016/j.rasd.2014.02.001.

Cosgrove, V. E., Rhee, S. H., Gelhorn, H. L., Boeldt, D., Corley, R. C., Ehringer, M. A., et al. (2011). Structure and etiology of cooccurring internalizing and externalizing disorders in adolescents. Journal of Abnormal Child Psychology, 39(1), 109-123.

Costa, N. M., Weems, C. F., Pellerin, K., \& Dalton, R. (2006). Parenting stress and childhood psychopathology: An examination of specificity to internalizing and externalizing symptoms. Journal of Psychopathology and Behavioral Assessment, 28(2), 113-122.

Craig, F., Operto, F. F., De Giacomo, A., Margari, L., Frolli, A., Conson, M., et al. (2016). Parenting stress among parents of children with neurodevelopmental disorders. Psychiatry Research, 242, 121-129.

Crnic, K. A., Gaze, C., \& Hoffman, C. (2005). Cumulative parenting stress across the preschool period: relations to maternal parenting and child behaviour at age 5. Infant and Child Development, 14(2), 117-132.

D’Onofrio, B. M., Slutske, W. S., Turkheimer, E., et al. (2007). Intergenerational transmission of childhood conduct problems: A children of twins study. Archives of General Psychiatry, 64(7), 820-829.

de Bruin, E., Ferdinand, R., Meester, S., de Nijs, P., \& Verheij, F. (2007). High rates of psychiatric co-morbidity in PDD-NOS. Journal of Autism and Developmental Disorders, 37(5), 877-886.

Deater-Deckard, K. (2004). Parenting stress. New Haven, CT: Yale University Press.

Duval, S., \& Tweedie, R. (2000). Trim and fill: A simple funnel-plotbased method of testing and adjusting for publication bias in meta-analysis. Biometrics, 56(2), 455-463.

Dykens, E. M., \& Lambert, W. (2013). Trajectories of diurnal cortisol in mothers of children with autism and other developmental disabilities: Relations to health and mental health. Journal of Autism and Developmental Disorders, 43(10), 2426-2434.

Egger, M., Davey Smith, G., Schneider, M., \& Minder, C. (1997). Bias in meta-analysis detected by a simple, graphical test. British Medical Journal, 315(7109), 629-634.

Eley, T., McAdams, T. A., Rijsdijk, F., Lichtenstein, P., Narusyte, J., Reiss, D., et al. (2015). The intergenerational transmission of anxiety: A children-of-twins study. American Journal of Psychiatry, 172(7), 630-637.

Estes, A., Munson, J., Dawson, G., Koehler, E., Zhou, X. H., \& Abbott, R. (2009). Parenting stress and psychological functioning among 
mothers of preschool children with autism and developmental delay. Autism, 13(4), 375-387.

Falk, N. H., Norris, K., \& Quinn, M. G. (2014). The factors predicting stress, anxiety and depression in the parents of children with autism. Journal of Autism and Developmental Disorders, 44(12), 3185-3203.

Farmer, C., Lecavalier, L., Yu, S., Arnold, L. E., McDougle, C. J., Scahill, L., et al. (2012). Predictors and moderators of parent training efficacy in a sample of children with autism spectrum disorders and serious behavioral problems. Journal of Autism and Developmental Disorders, 42(6), 1037-1044.

Firth, I., \& Dryer, R. (2013). The predictors of distress in parents of children with autism spectrum disorder. Journal of Intellectual and Developmental Disability, 38(2), 163-171.

Fitzgerald, M., Birkbeck, G., \& Matthews, P. (2002). Maternal burden in families with children with autistic spectrum disorder. Irish Journal of Psychological Medicine, 33(1), 2-12.

Foody, C., James, J. E., \& Leader, G. (2014). Parenting stress, salivary biomarkers, and ambulatory blood pressure in mothers of children with autism spectrum disorders. Research in Autism Spectrum Disorders, 8(2), 99-110.

Ford, T., Goodman, R., \& Meltzer, H. (2004). The relative importance of child, family, school and neighbourhood correlates of childhood psychiatric disorder. Social Psychiatry and Psychiatric Epidemiology, 39(6), 487-496.

Fung, S., Lunsky, Y., \& Weiss, J. A. (2015). Depression in youth with autism spectrum disorder: The role of ASD vulnerabilities and family-environmental stressors. Journal of Mental Health Research in Intellectual Disabilities, 8(3-4), 120-139.

Gallagher, S., Phillips, A. C., Oliver, C., \& Carroll, D. (2008). Predictors of psychological morbidity in parents of children with intellectual disabilities. Journal of Pediatric Psychology, 33(10), 1129-1136.

Giovagnoli, G., Postorino, V., Fatta, L. M., Sanges, V., De Peppo, L., Vassena, L., et al. (2015). Behavioral and emotional profile and parental stress in preschool children with autism spectrum disorder. Research in Developmental Disabilities, 45-46, 411-421.

Glennon, J. M., Karmiloff-Smith, A., \& Thomas, M. S. C. (2017). Syndromic autism: Progressing beyond current levels of description. Review Journal of Autism and Developmental Disorders, 4(4), 321-327.

Goodman, S. H., Rouse, M. H., Connell, A. M., Broth, M. R., Hall, C. M., \& Heyward, D. (2011). Maternal Depression and Child Psychopathology: A Meta-Analytic Review. Clinical Child and Family Psychology Review, 14(1), 1-27.

Hall, H. R., \& Graff, J. C. (2012). Maladaptive behaviors of children with autism: Parent support, stress, and coping. Issues in Comprehensive Pediatric Nursing, 35(3-4), 194-214.

Hastings, R. P., \& Brown, T. (2002). Behavior problems of children with autism, parental self-efficacy, and mental health. American Journal on Mental Retardation, 107(3), 222-232.

Hastings, R. P., Kovshoff, H., Ward, N. J., degli Espinosa, F., Brown, T., \& Remington, B. (2005). Systems analysis of stress and positive perceptions in mothers and fathers of pre-school children with autism. Journal of Autism and Developmental Disorders, 35(5), 635-644.

Hastings, R. P., Petalas, M. A., Jones, L., \& Totsika, V. (2014). Systems analysis of associations over time between maternal and sibling well-being and behavioral and emotional problems of children with autism. Research in Autism Spectrum Disorders, 8(11), 1516-1520.

Hayes, S. A., \& Watson, S. L. (2013). The impact of parenting stress: A meta-analysis of studies comparing the experience of parenting stress in parents of children with and without autism spectrum disorder. Journal of Autism and Developmental Disorders, 43(3), 629-642.

Herring, S., Gray, K., Taffe, J., Tonge, B., Sweeney, D., \& Einfeld, S. (2006). Behaviour and emotional problems in toddlers with pervasive developmental disorders and developmental delay: associations with parental mental health and family functioning. Journal of Intellectual Disability Research, 50, 874-882.

Higgins, J. P. T., Thompson, S. G., Deeks, J. J., \& Altman, D. G. (2003). Measuring inconsistency in meta-analyses. British Medical Journal, 327(7414), 557-560.

Huang, C.-Y., Yen, H.-C., Tseng, M.-H., Tung, L.-C., Chen, Y.-D., \& Chen, K.-L. (2014). Impacts of autistic behaviors, emotional and behavioral problems on parenting stress in caregivers of children with autism. Journal of Autism and Developmental Disorders, 44(6), 1383-1390.

Jellett, R., Wood, C. E., Giallo, R., \& Seymour, M. (2015). Family functioning and behaviour problems in children with autism spectrum disorders: The mediating role of parent mental health. Clinical Psychologist, 19(1), 39-48.

Jokiranta, E., Brown, A. S., Heinimaa, M., Cheslack-Postava, K., Suominen, A., \& Sourander, A. (2013). Parental psychiatric disorders and autism spectrum disorders. Psychiatry Research, 207(3), 203-211.

Jones, L., Hastings, R. P., Totsika, V., Keane, L., \& Rhule, N. (2014). Child behavior problems and parental well-being in families of children with autism: The mediating role of mindfulness and acceptance. American Journal on Intellectual and Developmental Disabilities, 119(2), 171-185.

Kerns, C. M., Kendall, P. C., Zickgraf, H., Franklin, M. E., Miller, J., \& Herrington, J. (2015). Not to be overshadowed or overlooked: functional impairments associated with comorbid anxiety disorders in youth with ASD. Behavior Therapy, 46(1), 29-39.

Keyser, D., Ahn, H., \& Unick, J. (2017). Predictors of behavioral problems in young children 3 to 9 years old: The role of maternal and child factors. Children and Youth Services Review, 82(Supplement C), 149-155.

Kim, I., Ekas, N. V., \& Hock, R. (2016). Associations between child behavior problems, family management, and depressive symptoms for mothers of children with autism spectrum disorder. Research in Autism Spectrum Disorders, 26, 80-90.

Kraemer, H. C., Stice, E., Kazdin, A., Offord, D., \& Kupfer, D. (2001). How do risk factors work together? Mediators, moderators, and independent, overlapping, and proxy risk factors. American Journal of Psychiatry, 158(6), 848-856.

Krakovich, T. M., McGrew, J. H., Yu, Y., \& Ruble, L. A. (2016). Stress in parents of children with autism spectrum disorder: An exploration of demands and resources. Journal of Autism and Developmental Disorders, 46(6), 2042-2053.

Kring, S. R., Greenberg, J. S., \& Seltzer, M. M. (2008). Adolescents and adults with autism with and without co-morbid psychiatric disorders: Differences in maternal well-being. Journal of Mental Health Research in Intellectual Disabilities, 1(2), 53-74.

Lancaster, R. L., Balling, K., Hastings, R., \& Lloyd, T. J. (2014). Attributions, criticism and warmth in mothers of children with intellectual disability and challenging behaviour: a pilot study. Journal of Intellectual Disability Research, 58(11), 1060-1071.

Lecavalier, L., Leone, S., \& Wiltz, J. (2006). The impact of behaviour problems on caregiver stress in young people with autism spectrum disorders. Journal of Intellectual Disability Research, 50, $172-183$.

Lee, J. K., \& Chiang, H. M. (2017). Parenting stress in South Korean mothers of adolescent children with autism spectrum disorder. International Journal of Developmental Disabilities, 64(2), 120-127.

Lovell, B., \& Wetherell, M. A. (2016). Behaviour problems of children with ASD and perceived stress in their caregivers: The 
moderating role of trait emotional intelligence? Research in Autism Spectrum Disorders, 28, 1-6.

Machado, S. B., Celestino, M. I. O., Serra, J. P. C., Caron, J., \& Ponde, M. P. (2016). Risk and protective factors for symptoms of anxiety and depression in parents of children with autism spectrum disorder. Developmental Neurorehabilitation, 19(3), 146-153.

Manning, M. M., Wainwright, L., \& Bennett, J. (2011). The double ABCX model of adaptation in racially diverse families with a school-age child with autism. Journal of Autism and Developmental Disorders, 41(3), 320-331.

McAdams, T. A., Rijsdijk, F. V., Neiderhiser, J. M., Narusyte, J., Shaw, D. S., Natsuaki, M. N., et al. (2015). The relationship between parental depressive symptoms and offspring psychopathology: Evidence from a children-of-twins study and an adoption study. Psychological Medicine, 45(12), 2583-2594.

McStay, R. L., Dissanayake, C., Scheeren, A., Koot, H. M., \& Begeer, S. (2014a). Parenting stress and autism: The role of age, autism severity, quality of life and problem behaviour of children and adolescents with autism. Autism, 18(5), 502-510.

McStay, R. L., Trembath, D., \& Dissanayake, C. (2014b). Stress and family quality of life in parents of children with autism spectrum disorder: Parent gender and the double ABCX model. Journal of Autism and Developmental Disorders, 44(12), 3101-3118.

Najman, J. M., Williams, G. M., Nikles, J., Spence, S., Bor, W., O'Callaghan, M., et al. (2000). Mothers' mental illness and child behavior problems: Cause-effect association or observation bias? Journal of the American Academy of Child \& Adolescent Psychiatry, 39(5), 592-602.

Neece, C. L., Green, S. A., \& Baker, B. L. (2012). Parenting stress and child behavior problems: A transactional relationship across time. American Journal on Intellectual and Developmental Disabilities, 117(1), 48-66.

Nicholson, J. S., Deboeck, P. R., Farris, J. R., Boker, S. M., \& Borkowski, J. G. (2011). Maternal depressive symptomatology and child behavior: transactional relationship with simultaneous bidirectional coupling. Developmental Psychology, 47(5), 1312-1323.

Osborne, L. A., McHugh, L., Saunders, J., \& Reed, P. (2008). The effect of parenting behaviors on subsequent child behavior problems in autistic spectrum conditions. Research in Autism Spectrum Disorders, 2(2), 249-263.

Osborne, L. A., \& Reed, P. (2009). The relationship between parenting stress and behavior problems of children with autistic spectrum disorders. Exceptional Children, 76(1), 54-73.

Pakenham, K. I., Samios, C., \& Sofronoff, K. (2005). Adjustment in mothers of children with Asperger syndrome: An application of the double ABCX model of family adjustment. Autism, 9(2), 191-212.

Park, S., Park, M. H., Kim, H. J., \& Yoo, H. J. (2013). Anxiety and depression symptoms in children with Asperger syndrome compared with attention-deficit/hyperactivity disorder and depressive disorder. Journal of Child and Family Studies, 22(4), 559-568.

Paynter, J., Riley, E., Beamish, W., Davies, M., \& Milford, T. (2013). The double ABCX model of family adaptation in families of a child with an autism spectrum disorder attending an Australian early intervention service. Research in Autism Spectrum Disorders, 7(10), 1183-1195.

Peters, J. L., Sutton, A. J., Jones, D. R., Abrams, K. R., Rushton, L., \& Moreno, S. G. (2010). Assessing publication bias in metaanalyses in the presence of between-study heterogeneity. Journal of the Royal Statistical Society: Series A (Statistics in Society), 173(3), 575-591.

Peters-Scheffer, N., Didden, R., \& Korzilius, H. (2012). Maternal stress predicted by characteristics of children with autism spectrum disorder and intellectual disability. Research in Autism Spectrum Disorders, 6(2), 696-706.
Podsakoff, P. M., MacKenzie, S. B., Lee, J. Y., \& Podsakoff, N. P. (2003). Common method biases in behavioral research: A critical review of the literature and recommended remedies. Journal of Applied Psychology, 88(5), 879-903.

Pozo, P., Sarria, E., \& Brioso, A. (2014). Family quality of life and psychological well-being in parents of children with autism spectrum disorders: A double ABCX model. Journal of Intellectual Disability Research, 58(5), 442-458.

R Core Team. (2013). R: A language and environment for statistical computing. R Foundation for Statistical Computing, Vienna, Austria. Retrieved from http://www.R-project.org/.

Reaven, J., Washington, L., Moody, E. J., Stern, J. A., Hepburn, S. L., \& Blakeley-Smith, A. (2015). Examining the relationship between parental anxiety and treatment response in children and adolescents with autism spectrum disorder and anxiety. Journal of Autism and Developmental Disorders, 45(8), 2464-2473.

Reed, P., Howse, J., Ho, B., \& Osborne, L. A. (2016). Relationship between perceived limit-setting abilities, autism spectrum disorder severity, behaviour problems and parenting stress in mothers of children with autism spectrum disorder. Autism, 28, 28.

Reed, P., Osborne, L. A., Makrygianni, M., Waddington, E., Etherington, A., \& Gainsborough, J. (2013). Evaluation of the Barnet Early Autism Model (BEAM) teaching intervention programme in a 'real world' setting. Research in Autism Spectrum Disorders, 7(6), 631-638.

Rezendes, D. L., \& Scarpa, A. (2011). Associations between parental anxiety/depression and child behavior problems related to autism spectrum disorders: The roles of parenting stress and parenting self-efficacy. Autism Research and Treatment, 2011, 395190.

Robinson, M., \& Neece, C. L. (2015). Marital satisfaction, parental stress, and child behavior problems among parents of young children with developmental delays. Journal of Mental Health Research in Intellectual Disabilities, 8(1), 23-46.

Rosenthal, R. (1979). The file drawer problem and tolerance for null results. Psychological Bulletin, 86(3), 638-641.

Salazar, F., Baird, G., Chandler, S., Tseng, E., O’Sullivan, T., Howlin, P., et al. (2015). Co-occurring psychiatric disorders in preschool and elementary school-aged children with autism spectrum disorder. Journal of Autism and Developmental Disorders, 45(8), 2283-2294.

Sawyer, M. G., Bittman, M., La Greca, A. M., Crettenden, A. D., Harchak, T. F., \& Martin, J. (2010). Time Demands of Caring for Children with Autism: What are the Implications for Maternal Mental Health? Journal of Autism and Developmental Disorders, 40(5), 620-628.

Shawler, P. M., \& Sullivan, M. A. (2017). Parental stress, discipline strategies, and child behavior problems in families with young children with autism spectrum disorders. Focus on Autism and Other Developmental Disabilities, 32(2), 142-151.

Simonoff, E., Jones, C. R., Baird, G., Pickles, A., Happe, F., \& Charman, T. (2013). The persistence and stability of psychiatric problems in adolescents with autism spectrum disorders. Journal of Child Psychology and Psychiatry and Allied Disciplines, 54(2), 186-194.

Simonoff, E., Pickles, A., Charman, T., Chandler, S., Loucas, T., \& Baird, G. (2008). Psychiatric disorders in children with autism spectrum disorders: Prevalence, comorbidity, and associated factors in a population-derived sample. Journal of the American Academy of Child and Adolescent Psychiatry, 47(8), 921-929.

Skokauskas, N., \& Gallagher, L. (2012). Mental health aspects of autistic spectrum disorders in children. Journal of Intellectual Disability Research, 56(3), 248-257.

Stone, L. L., Mares, S. H. W., Otten, R., Engels, R., \& Janssens, J. (2016). The co-development of parenting stress and childhood 
internalizing and externalizing problems. Journal of Psychopathology and Behavioral Assessment, 38(1), 76-86.

Stoppelbein, L., Biasini, F., Pennick, M., \& Greening, L. (2016). Predicting internalizing and externalizing symptoms among children diagnosed with an autism spectrum disorder: The role of routines. Journal of Child and Family Studies, 25(1), 251-261.

Stratis, E. A., \& Lecavalier, L. (2015). Informant agreement for youth with autism spectrum disorder or intellectual disability: A metaanalysis. Journal of Autism and Developmental Disorders, 45(4), 1026-1041.

Suzuki, K., Kobayashi, T., Moriyama, K., Kaga, M., Hiratani, M., Watanabe, K., et al. (2015). Development and evaluation of a parenting resilience elements questionnaire (PREQ) measuring resiliency in rearing children with developmental disorders. PLOS ONE, 10(12), e0143946

Taylor, J. L., \& Warren, Z. E. (2012). Maternal depressive symptoms following autism spectrum diagnosis. Journal of Autism and Developmental Disorders, 42(7), 1411-1418.

Totsika, V., Hastings, R. P., Emerson, E., Lancaster, G. A., Berridge, D. M., \& Vagenas, D. (2013). Is there a bidirectional relationship between maternal well-being and child behavior problems in autism spectrum disorders? Longitudinal analysis of a population-defined sample of young children. Autism Research, 6(3), 201-211.

Valicenti-McDermott, M., Lawson, K., Hottinger, K., Seijo, R., Schechtman, M., Shulman, L., et al. (2015). Parental stress in families of children with autism and other developmental disabilities. Journal of Child Neurology, 30(13), 1728-1735.

Vasilopoulou, E., \& Nisbet, J. (2016). The quality of life of parents of children with autism spectrum disorder: A systematic review. Research in Autism Spectrum Disorders, 23, 36-49.

Viechtbauer, W. (2010). Conducting meta-analyses in R with the metafor package. Journal of Statistical Software, 36(3), 48.

Walsh, C. E., Mulder, E., \& Tudor, M. E. (2013). Predictors of parent stress in a sample of children with ASD: Pain, problem behavior, and parental coping. Research in Autism Spectrum Disorders, $7(2), 256-264$.

Warfield, M. E., Chiri, G., Leutz, W. N., \& Timberlake, M. (2014). Family well-being in a participant-directed autism waiver program: The role of relational coordination. Journal of Intellectual Disability Research, 58(12), 1091-1104.

Weiss, J. A., Cappadocia, M., MacMullin, J. A., Viecili, M., \& Lunsky, Y. (2012). The impact of child problem behaviors of children with ASD on parent mental health: The mediating role of acceptance and empowerment. Autism, 16(3), 261-274.

Weiss, J. A., Cappadocia, M. C., Tint, A., \& Pepler, D. (2015). Bullying victimization, parenting stress, and anxiety among adolescents and young adults with autism spectrum disorder. Autism Research, 8(6), 727-737.

Weitlauf, A. S., Vehorn, A. C., Taylor, J. L., \& Warren, Z. E. (2014). Relationship satisfaction, parenting stress, and depression in mothers of children with autism. Autism, 18(2), 194-198.

Wilson, D. B. (2001). Practical meta-analysis effect size calculator. https://www.campbellcollaboration.org/escalc/html/EffectSize Calculator-Home.php. Accessed 10 October 20172017.

Zadeh, Z. Y., Jenkins, J., \& Pepler, D. (2010). A transactional analysis of maternal negativity and child externalizing behavior. International Journal of Behavioral Development, 34(3), 218-228.

Zaidman-Zait, A., Mirenda, P., Duku, E., Szatmari, P., Georgiades, S., Volden, J., et al. (2014). Examination of bidirectional relationships between parent stress and two types of problem behavior in children with autism spectrum disorder. Journal of Autism and Developmental Disorders, 44(8), 1908-1917.

Zaidman-Zait, A., Mirenda, P., Duku, E., Vaillancourt, T., Smith, I. M., Szatmari, P., et al. (2017). Impact of personal and social resources on parenting stress in mothers of children with autism spectrum disorder. Autism, 21(2), 155-166. 